\title{
Bile Acids in Neurodegenerative Disorders
}

\author{
Hayley D. Ackerman and Glenn S. Gerhard* \\ Department of Medical Genetics and Molecular Biochemistry, The Lewis Katz School of Medicine at Temple University, \\ Philadelphia, PA, USA
}

Bile acids, a structurally related group of molecules derived from cholesterol, have a long history as therapeutic agents in medicine, from treatment for primarily ocular diseases in ancient Chinese medicine to modern day use as approved drugs for certain liver diseases. Despite evidence supporting a neuroprotective role in a diverse spectrum of age-related neurodegenerative disorders, including several small pilot clinical trials, little is known about their molecular mechanisms or their physiological roles in the nervous system. We review the data reported for their use as treatments for neurodegenerative diseases and their underlying molecular basis. While data from cellular and animal models and clinical trials support potential efficacy to treat a variety of neurodegenerative disorders, the relevant bile acids, their origin, and the precise molecular mechanism(s) by which they confer neuroprotection are not known delaying translation to the clinical setting.

Keywords: bile acids, neuroprotection, neurodegeneration

OPEN ACCESS

Edited by:

Aurel Popa-Wagner, University of Rostock, Germany

Reviewed by:

Ralf J. Braun

University of Bayreuth, Germany

Michael Lardelli,

University of Adelaide, Australia

Raluca Sandu Vintilescu, University of Medicine and Pharmacy

of Craiova, Romania

*Correspondence:

Glenn S. Gerhard

gsgerhard@temple.edu

Received: 11 August 2016

Accepted: 21 October 2016

Published: 22 November 2016

Citation:

Ackerman HD and Gerhard GS (2016)

Bile Acids in Neurodegenerative

Disorders.

Front. Aging Neurosci. 8:263.

doi: 10.3389/fnagi.2016.00263

\section{BACKGROUND}

Bile acids are a structurally related group of molecules derived from cholesterol that are widely known for their role as chemical detergents involved in the intestinal absorption and transport of fats and lipid-soluble nutrients (Schonewille et al., 2016). However, bile acids also appear to play as yet poorly defined physiological roles in the central nervous system (Lieu et al., 2014). Surprisingly little work has been done on the physiological roles of bile acids in neurons or the central nervous system (Zhang et al., 1997) despite a wide array of data in model systems and despite the significant therapeutic advantages of bile acids. Bile acids are readily bioavailable via oral, subcutaneous, or intravenous administration, can cross the blood-brain barrier, are relatively nontoxic, and have been approved by the U.S. Food and Drug Administration for human therapeutic use. We review evidence supporting a potentially therapeutic role for bile acids in a number of diverse neurodegenerative conditions. A summary of the studies described below is presented in Tables 1, 2.

\section{BIOLOGY OF BILE ACIDS}

Two aspects of bile acid metabolism are relevant to their role in neurodegenerative disorders, bile acids that circulate systemically and that are synthesized by neurons. Circulating bile acids are largely synthesized from cholesterol in the liver (Prawitt et al., 2011). Ingestion of food causes bile acid secretion from the gallbladder through the common bile duct to the duodenum in order to facilitate the absorption of lipids and lipid-soluble vitamins via formation of micelles. Upon reaching the ileum, bile acids are transported by specific transport proteins to the portal circulation 


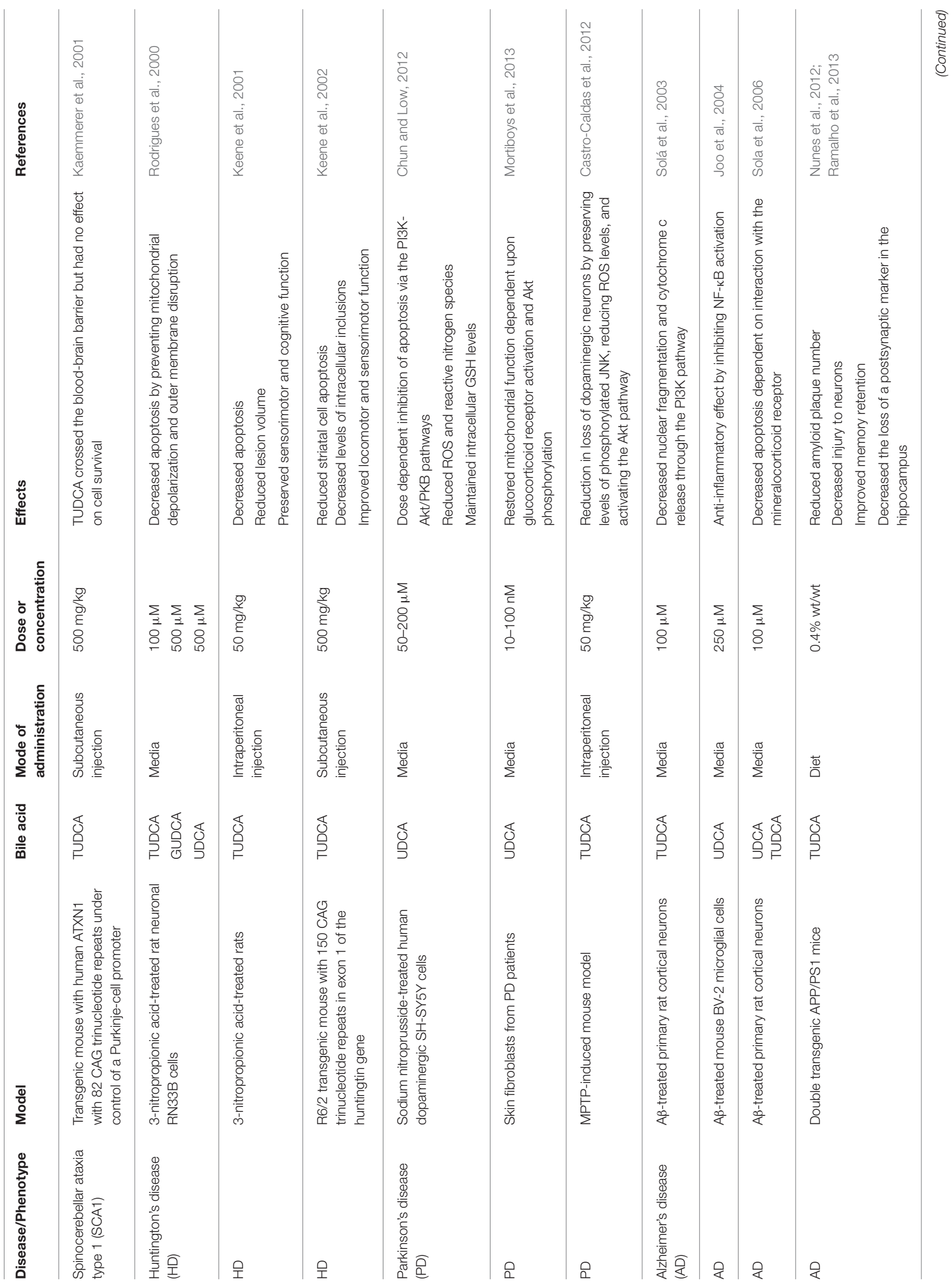




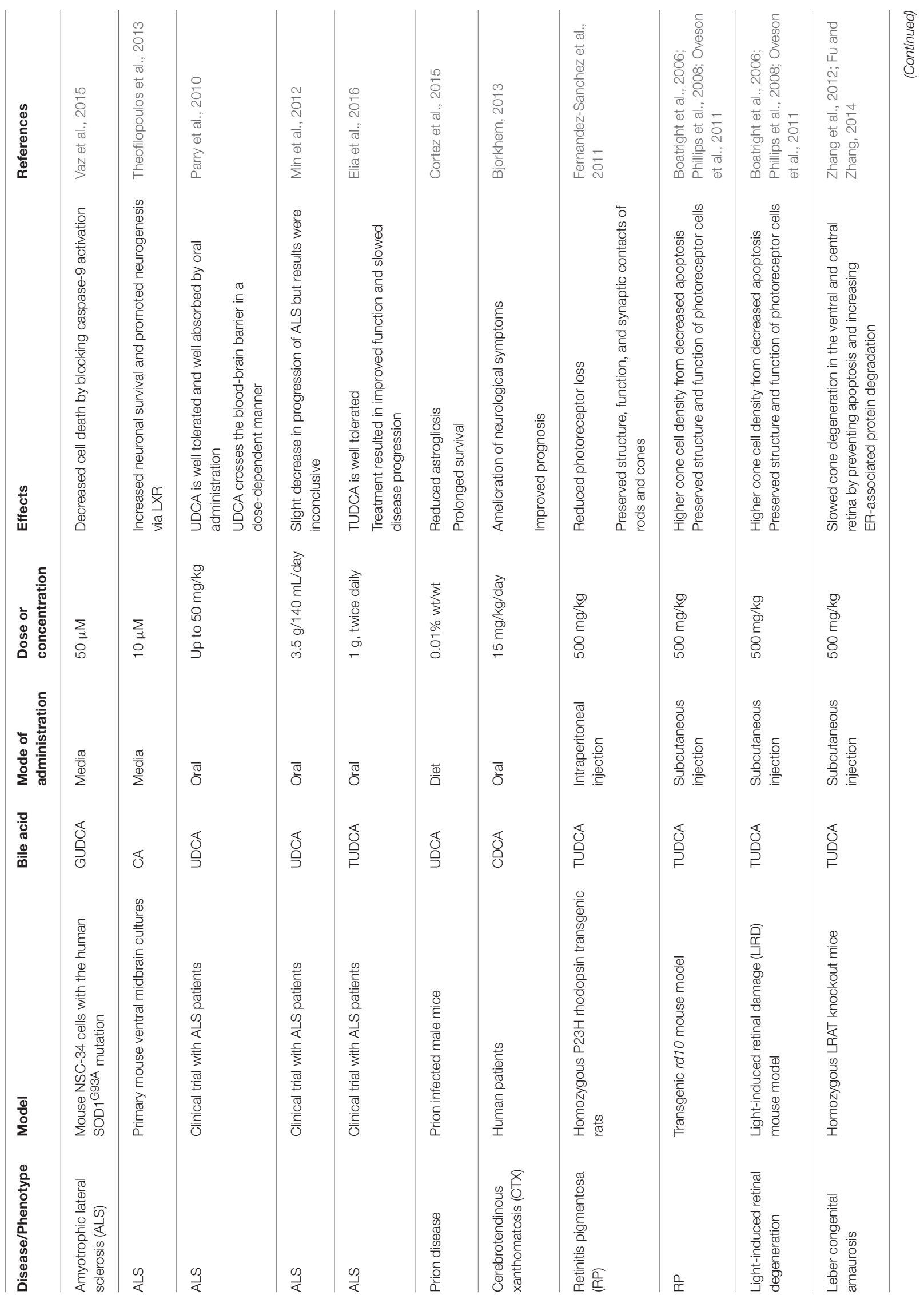




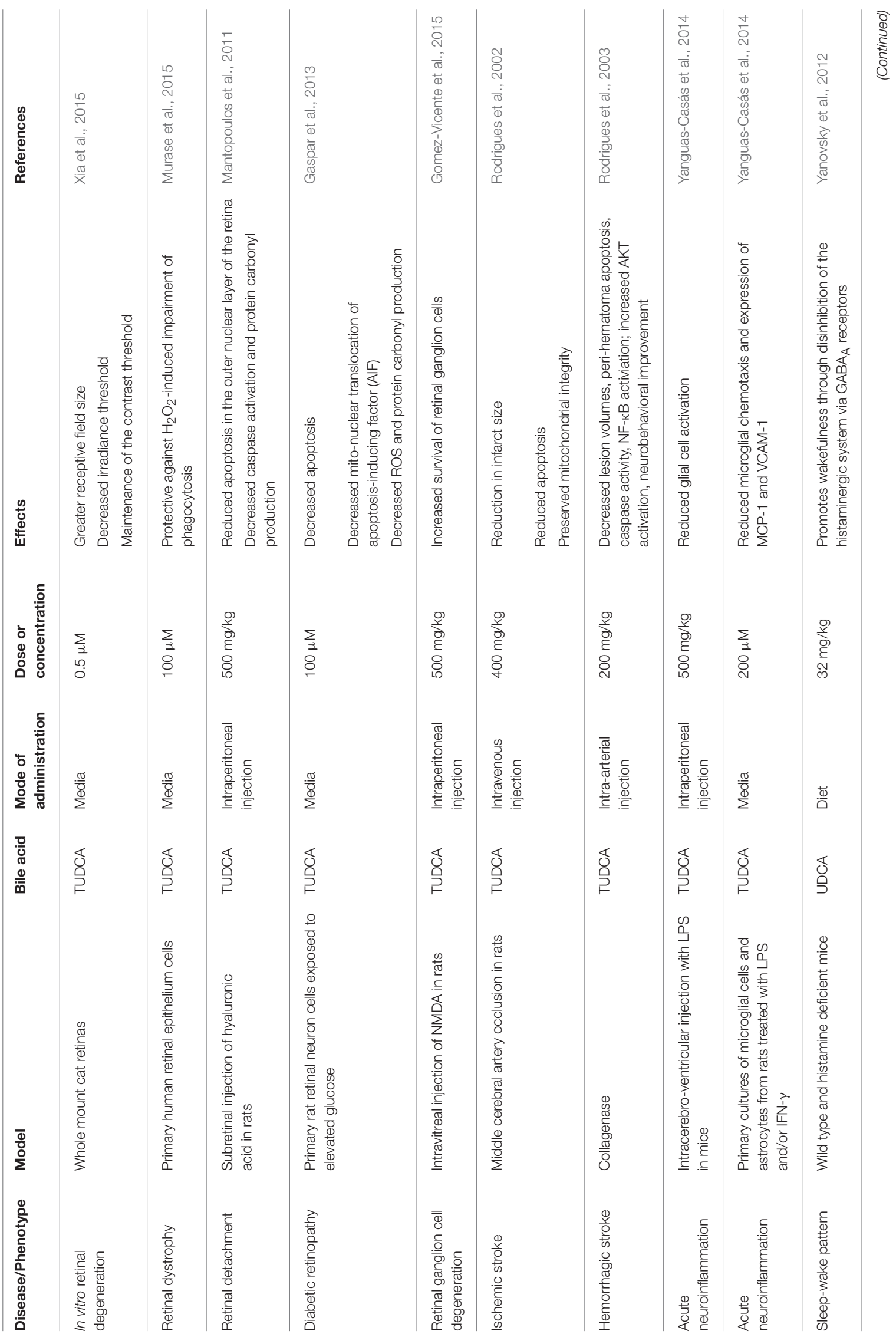


for recycling back to the liver. The process is highly efficient with over $95 \%$ of bile acids resorbed, the remaining 5\% proceeding to the colon and excreted through the stool. Enterohepatic recycling of the bile acid pool occurs about 12 times per day, thus the net flux of bile acids through primarily the portal, but also the systemic, circulation is substantial.

The two primary bile acids produced by the liver in humans are cholic acid (CA) and chenodeoxycholic acid (CDCA). These primary bile acids can undergo conjugation with glycine or taurine prior to secretion in the bile to form glycocholic acid (GCA), taurocholic acid (TCA), glycochenodeoxycholic acid (GCDCA), and taurochenodeoxycholic acid (TCDCA). In the intestine, they can undergo dehydroxylation by gut bacteria to produce deoxycholic acid (DCA) and lithocholic acid (LCA). Further chemical modifications can also occur resulting in other minor species such as ursodeoxycholic acid (UDCA) (Zhang et al., 1997).

Bile acids also function as signaling molecules through interaction with several receptor systems (Figure 1). They serve as ligands for the nuclear transcription factor farnesoid $\mathrm{X}$ receptor (FXR), which forms a heterodimeric complex with retinoid $\mathrm{X}$ receptor $\alpha(\mathrm{RXR} \alpha)$ that binds to an inverted repeat sequence in gene promoters (Hoeke et al., 2014). They are also agonists for a cellular receptor, the $\mathrm{G}$ protein-coupled bile acid receptor 1 (GPBAR1 or TGR5), to mediate signaling via the generation of cyclic adenosine monophosphate (cAMP) by adenylate cyclase, which stimulates cAMP-dependent protein kinase $\mathrm{A}$ (PKA) and phosphorylation of the cAMP response element binding protein (CREB) transcription factor (Hodge and Nunez, 2016; Schonewille et al., 2016).

\section{ALZHEIMER'S DISEASE}

Alzheimer's disease (AD) is characterized by dementia and memory loss that is associated with extracellular senile plaques of amyloid precursor protein derived amyloid-beta $(A \beta)$ protein and intracellular neurofibrillary tangles consisting of the Tau protein (Scheltens et al., 2016). A primary in vitro model of $\mathrm{AD}$ is therefore treatment of cells with $\mathrm{A} \beta$ peptide that causes cytotoxicity to both primary cultures of neurons as well as neuronal cell lines. Treatment of primary cortical neuron cultures derived from Wistar rat embryos with tauroursodeoxycholic acid (TUDCA) prior to exposure to $A \beta$ peptide significantly decreased nuclear fragmentation and cytochrome $c$ release that was dependent upon activation of the PI3K pathway (Solá et al., 2003). The bile acid UDCA was found to regulate IкB $\alpha$ and NF$\kappa B$ regulated genes in the mechanism of protection against $A \beta$ toxicity in the BV-2 microglial cell line (Joo et al., 2004).

Certain steroid hormones exhibit neuroprotective properties that are thought to be mediated in part through interaction with nuclear steroid receptors, including the glucocorticoid, and mineralocorticoid receptors (Garcia-Segura and Balthazart, 2009). Neurosteroids and bile acids are cholesterol derivatives that share some structural similarity so certain bile acids are natural ligands for these receptors. UDCA and TUDCA reduced $\mathrm{A} \beta$ induced apoptosis of primary rat cortical neurons that was 
TABLE 2 | Genomic and metabolomics data implicating bile acids in neurodegenerative disorders.

\begin{tabular}{|c|c|c|c|}
\hline Disease & Approach & Genetic association & References \\
\hline PD & Meta-analysis of GWAS data from PD and normal patients & HSD3B7 missense SNP in HSD3B7, & $\begin{array}{l}\text { Cheng et al., 2003; Song } \\
\text { and Lee, } 2013\end{array}$ \\
\hline PD & Meta-analysis of PD miRNA GWAS data & SNPs in a miRNA-binding site in the 3' UTR of HSD3B7 & Ghanbari et al., 2016 \\
\hline ALS & Peripheral blood cell eQTL of ALS and normal patients & CYP27A1 eQTL & Diekstra et al., 2012 \\
\hline$A D$ & Plasma metabolomic analysis of AD and normal patients & $\begin{array}{l}\text { Increased plasma GUDCA levels in patients with mild } \\
\text { cognitive impairment or AD }\end{array}$ & Mapstone et al., 2014 \\
\hline
\end{tabular}

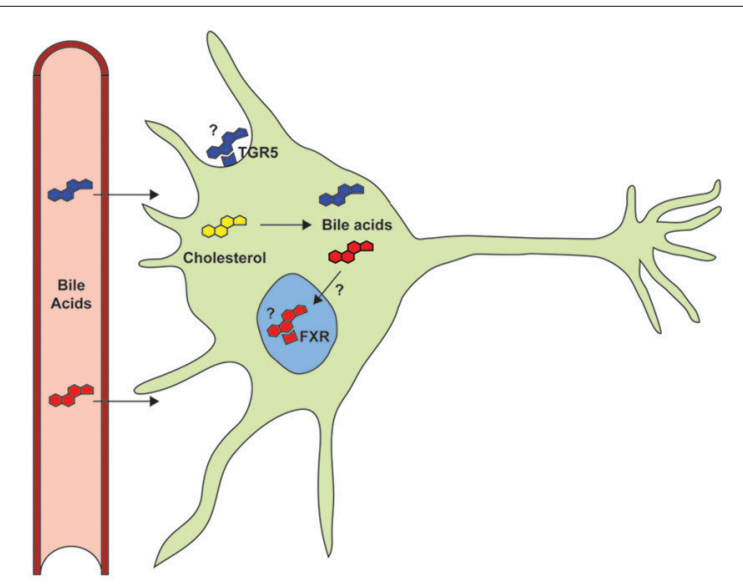

FIGURE 1 | Despite documented neuroprotective roles in models of neurodegenerative disorders, the primary signaling pathways (TGR5 and FXR) and the potential role of endogenous bile acids have not yet been studied.

found to be dependent upon the mineralocorticoid but not the glucocorticoid receptor (Sola et al., 2006). TUDCA appeared to interact with the ligand binding domain of the mineralocorticoid receptor to prevent its binding to heat shock protein 90 and subsequent trafficking for proteosomal processing allowing for translocation to the nucleus.

Bile acids have also been studied in animal models of AD. In double transgenic mice (APP/PS1) that express human amyloid precursor protein carrying the KM670/671NL Swedish double mutation and the human presenilin 1 L166P mutation under the regulation of a neuron-specific promoter, treatment via dietary supplementation of standard laboratory chow with $0.4 \%$ (wt/wt) of TUDCA significantly reduced amyloid plaque number in the frontal cortex and hippocampus, decreased injury to neurons measured by determining loss of, or damage to, neuronal fibers surrounding plaques, and improved memory retention measured via contextual, though not auditory, fear conditioning (Nunes et al., 2012) as well as reduced hippocampal and prefrontal amyloid deposition (Lo et al., 2013). TUDCA has also been shown to help preserve the postsynaptic marker postsynaptic density-95 in the hippocampus of APP/PS1 mice (Ramalho et al., 2013). Repurposed RXR agonists or "rexinoids" have also shown some effectiveness in transgenic models of $\mathrm{AD}$ (Koster et al., 2016), suggesting a possible involvement of bile acids.
In humans, a recent study of cognitively intact patients identified and validated a set of blood-based biomarkers that included glycoursodeoxycholic acid (GUDCA) that could predict the onset of either AD or amnestic mild cognitive impairment, considered an early precursor of $\mathrm{AD}$, within 2-3 years with an accuracy of over $90 \%$ (Mapstone et al., 2014). This suggests a potential association of bile acids in the progression or preclinical neurodegenerative phase of $\mathrm{AD}$.

\section{PARKINSON'S DISEASE}

The effects of bile acids on chemical and genetic models of Parkinson's disease (PD), which is characterized by the selective loss of dopaminergic neurons in the substantia nigra region of the brain and a resulting tremor, have been reported. Sodium nitroprusside (SNP)-induced cytotoxicity of human dopaminergic SH-SY5Y cells has been used as a model of PD. UDCA was found to dose-dependently (50-200 $\mu \mathrm{M})$ decrease SNP-related cell death. UDCA reduced reactive oxygen species (ROS), reactive nitrogen species (peroxynitrite and nitric oxide), and helped to maintain intracellular glutathione (GSH) levels. Apoptosis markers including nuclear fragmentation, caspase activation, and cytochrome c release were correspondingly reduced. Inhibiting phosphatidylinositiol-3-kinase (PI3K) and Akt/PKB blocked the favorable effects of UDCA on SNP-induced cytotoxic cell death (Chun and Low, 2012).

Bile acids have also been implicated in PD through genetic association studies. In a meta-analysis of genome-wide association study (GWAS) data based on the genotypes of 2,525,705 SNPs in 4238 PD cases and 4239 non-PD controls, a total of 3 SNPs were found to be statistically associated with $\mathrm{PD}$, including a non-synonymous missense variant in HSD3B7 (hydroxy-delta-5-steroid dehydrogenase, 3 beta- and steroid delta-isomerase 7) (Song and Lee, 2013). Recessive mutations in HSD3B7, which catalyzes the second step in the classical pathway of bile acid synthesis, are associated with loss of bile acid synthetic capability and progressive liver disease (Cheng et al., 2003). In a GWAS of 48,844 SNPs residing in miRNA-binding site variants, 32 SNPs were associated with PD that were located in the $3^{\prime}$ untranslated regions of 13 genes including HSD3B7 (Ghanbari et al., 2016), providing further genetic evidence for a role of bile acids in PD.

Mitochondrial dysfunction has been associated with PD (Luo et al., 2015). To identify compounds that could restore mitochondrial function in skin fibroblasts obtained from patients with a PD parkin (PARK2) gene mutation, 
a 2000 compound library was screened for significant improvement in mitochondrial membrane potential (Mortiboys et al., 2013). Ursocholanic acid and the related compound dehydro(11,12)ursolic acid lactone were among the top 15 compounds that had dose response characteristics favorable for drug development and lacked many of the disadvantages of the other top hits. The structurally related bile acid UDCA was also found to rescue mitochondrial function to a similar extent, which was dependent upon activation of the glucocorticoid receptor and increased phosphorylation of Akt. UDCA was also found to restore mitochondrial function in fibroblasts obtained from a PD patient with a LRRK2-G2019S mutation.

The 1-methyl-4-phenyl-1,2,3,6-tetrahydropyridine (MPTP) neurotoxin is a widely used toxin model for PD, replicating most of the clinical and pathological features of PD in humans and animal models. TUDCA was found to play a role in ameliorating neurodegeneration in MPTP-induced degeneration of dopaminergic neurons in the nigrostriatal axis in C57BL/6 glutathione S-transferase pi (GSTP) null mice (Castro-Caldas et al., 2012). Treatment of mice with TUDCA prior to MPTP caused a $30 \%$ reduction in loss of dopaminergic neurons and reduced dopaminergic fiber loss. MPTP toxicity has also been associated with increased ROS production and activation of JNKmediated apoptosis (Huang et al., 2016). TUDCA reduced levels of ROS and preserved levels of phosphorylated JNK (p-JNK) (Castro-Caldas et al., 2012).

\section{AMYOTROPHIC LATERAL SCLEROSIS}

The loss of motor neurons in Amyotrophic lateral sclerosis (ALS) has prompted therapeutic strategies aimed at preventing neuronal cell death and promoting regeneration (Carvalho et al., 2015). NSC-34 cells, created by fusion of cultured neuroblastoma cells and motor neurons from mouse spinal cord, can be treated with retinoic acid to induce neurite outgrowth and functional characteristics of motor neurons and are considered a highly stable and widely used murine motor neuron cell line model (Maier et al., 2013; Veyrat-Durebex et al., 2014). NSC-34 cells modified to carry the human form of SOD1 with the G93A mutation (hSOD1 ${ }^{\mathrm{G} 93 \mathrm{~A}}$ ) have thus been used as a model for ALS. GUDCA was reported to reduce cell death in the NSC-34 hSOD1 ${ }^{\text {G93A }}$ cell model and to block caspase-9 activation (Vaz et al., 2015).

Bile acids also appear to have physiological roles in the central nervous system. CA, a bile acid present in the adult brain, was identified by LC/MS as a ligand of liver X receptor (LXR), activating LXR but not FXR in ventral midbrain dopaminergic neurons (Theofilopoulos et al., 2013). 6 $\alpha$-hydroxylated bile acids and the synthetic bile acid ligand GW3965 were also highly potent activators of LXR. Male LXR knockout mice develop an adult-onset motor neuron degeneration that is associated with impairment of motor coordination, axonal atrophy, astrogliosis, accumulation of lipid and loss of motor neurons in the spinal cord, findings similar to the neuropathology of ALS (Andersson et al., 2005). Additionally, FXR has been shown to contribute to normal motor function in mice (Huang et al., 2015).
Several clinical trials of bile acids have been conducted in ALS patients in which evidence for safety and potential efficacy was observed (Parry et al., 2010; Min et al., 2012; Elia et al., 2016). In an ALS clinical trial with orally administered UDCA, the bile acid was found to be well tolerated and crossed into the cerebrospinal fluid in a dose-dependent manner (Parry et al., 2010). In a separate clinical trial to test the efficacy of UDCA for treating ALS patients, oral solubilized administration for 3 months was shown to be well tolerated and there was a slight decrease in the progression of ALS in the treatment group as compared to the placebo group (Min et al., 2012). However, due to the small size of the study and the high rate of patient dropout, the efficacy of the treatment was inconclusive. TUDCA administered orally twice per day for over 1 year resulted in a higher percentage of subjects achieving at least a $15 \%$ improvement in the ALS Functional Rating Scale Revised (ALSFRS-R) slope (Elia et al., 2016).

Genetic data also implicates bile acid metabolism in ALS. Gene expression profiles obtained from the peripheral blood cells of sporadic ALS patients and normal controls were analyzed in the context of genome-wide SNP genotype data to identify expression quantitative trait loci (eQTLs). A cluster of transcriptSNP pairs with the highest level of statistical significance and meeting correction for multiple testing was associated with CYP27A1 expression in ALS (Diekstra et al., 2012). CYP27A1 is a key enzyme in the alternative bile acid synthesis pathway, and mutations in this enzyme can cause cerebrotendinous xanthomatosis as described below.

\section{HUNTINGTON'S DISEASE}

Huntington's disease (HD), an autosomal dominantly inherited neurodegenerative disease, is caused by an expansion mutation in CAG triplet repeat number in exon 1 of the huntingtin (HTT) gene (Ross et al., 2014). It is characterized by involuntary choreic movements, psychiatric and behavioral disturbances, and impaired cognitive function. Genetic and chemical animal and cellular models have been developed in which the effects of bile acids have been assessed. For example, 3-nitropropionic acid, an irreversible chemical inhibitor of mitochondrial succinate dehydrogenase, can be used to induce apoptosis of cells in culture and in neurons in the striatum region of the brain that causes changes similar to the morphology and neurochemical changes of HD. Incubation of rat neuronal RN33B cells with TUDCA was found to reduce 3-nitropropionic acid induced apoptosis by about $80 \%$ (Rodrigues et al., 2000). Other bile acids, including UDCA and GUDCA, were also associated with decreased apoptosis, preventing 3-nitropropionic acid induced release of mitochondrial cytochrome $c$, and associated morphological changes in both isolated mitochondria and in intact cells. In rats treated with TUDCA, a similar level of reduction in apoptosis and in the volume of lesions associated with administration of 3-nitropropionic acid was observed that was correlated with preservation of sensorimotor function and performance in cognitive task assays (Keene et al., 2001). These results suggest that the mechanisms of bile acid protection may be similar both in vitro and in vivo. 
Genetic models of HD have also been studied, including the R6/2 mouse, which is transgenic for a 150 repeat trinucleotide CAG expansion in exon 1 of HTT and manifests severe neurodegeneration, neuronal intranuclear inclusions, and sensorimotor deficits that result in a severely shortened survival of less than 4 months of age (Mangiarini et al., 1996; Davies et al., 1997). TUDCA treatment was administered to the R6/2 transgenic mice subcutaneously at a dose of 500 $\mathrm{mg} / \mathrm{kg}$ once every 3 days from weaning to 6 months of age, which was tolerated without skin reaction or other effects. The subcutaneously administered TUDCA was absorbed into the circulation, crossed the blood-brain barrier, and produced sevenfold increases in UDCA levels in the brain without evidence of adverse effects. TUDCA treatment resulted in significantly reduced striatal cell apoptosis, decreased levels of intracellular inclusions, and improved locomotor open field and sensorimotor Rota-Rod performances (Keene et al., 2002).

\section{SPINOCEREBELLAR ATAXIA TYPE 1}

Spinocerebellar ataxia type 1 (SCA1) is another dominantly inherited neurodegenerative disorder characterized chiefly by progressive ataxia (Meera et al., 2016). SCA1 is caused by expansion of an unstable CAG trinucleotide repeat that encodes a glutamine tract in the Ataxin-1 gene (ATXN1). The disorder is characterized primarily by degeneration of Purkinje neurons of the cerebellum. TUDCA treatment was used to determine whether the survival of Purkinje cells and the onset and progression of ataxia was altered in a transgenic mouse model of SCA1, in which 30 copies of the human ATXN1 cDNA containing a CAG trinucleotide repeat of 82 repeats was under the control of the Pcp-2 Purkinje-cell specific promoter 1 (Kaemmerer et al., 2001). Despite the successful administration of TUDCA using a similar subcutaneous injection protocol as described above for the R6/2 transgenic $\mathrm{HD}$ mouse, and documentation of increased levels in the brain, no effects on cell survival or on the neurological phenotype were noted. The disparate effects in two different genetic models of neurodegenerative disease, suggest that bile acids target specific pathways.

\section{PRION DISEASES}

Neurodegenerative disorders caused by prions include Creutzfeldt-Jakob disease and similar disorders in animals such as bovine spongiform encephalopathy in cows, chronic wasting disease in deer, and scrapie in sheep (Imran and Mahmood, 2011; Windl and Dawson, 2012). Prion protein $\left(\mathrm{PrP}^{\mathrm{C}}\right)$ is a normal cellular protein that when mutated becomes misfolded $\left(\mathrm{PrP}^{\mathrm{Sc}}\right)$. $\mathrm{PrP}^{\mathrm{Sc}}$ can then convert normally folded $\mathrm{PrP}^{\mathrm{C}}$ to the misfolded form. Accumulation of $\mathrm{PrP}^{\mathrm{Sc}}$ causes loss of neurons, astrogliosis, and spongiform degeneration resulting in dementia, ataxia, and death. One approach to therapeutic development has been to block or interfere with the conversion of $\mathrm{PrP}^{\mathrm{C}}$ to $\mathrm{PrP}^{\mathrm{Sc}}$. TUDCA and UDCA were found to substantially reduce this conversion in cell-free aggregation assays as well as in both chronically and acutely infected mouse ScN2a neuroblastoma cells (Cortez et al.,
2015). TUDCA and UDCA also reduced neuronal loss in a prion organotypic slice culture model of intracerebral infection that assesses prion replication occurring ex vivo through infection of brain slices with prion infected brain homogenate. UDCA treatment also reduced astrocytosis and prolonged survival in prion infected male C57BL/6 mice, although whether bile acids interacted with the $\operatorname{PrP}^{\mathrm{C}}$ to $\operatorname{PrP}^{\mathrm{Sc}}$ conversion or mediated protective effects through some other mechanism is not known.

\section{CEREBROTENDINOUS XANTHOMATOSIS}

Cerebrotendinous xanthomatosis (CTX) is a very rare autosomal recessive disorder caused by mutations in the CYP27A1 gene (Björkhem and Hansson, 2010; Bjorkhem, 2013). These mutations lead to deficiency of the sterol 27-hydroxylase inner mitochondrial membrane protein. Sterol 27-hydroxylase oxidizes cholesterol to 27-hydroxycholesterol in the alternative bile acid synthesis pathway that leads to the generation of CDCA. Sterol 27-hydroxylase deficiency leads to a reduction of CDCA and upregulation of cholesterol $7 \alpha$-hydroxylase (CYP7A1), the ratelimiting enzyme in the classic bile acid synthesis pathway resulting in elevated levels of cholestanol and bile alcohols. CTX patients have a mean age of diagnosis of 35 years and manifest multiple neurologic symptoms including dementia, ataxia, peripheral neuropathy, epilepsy, myopathy, and psychiatric disorders, as well as a variety of non-neurological manifestations including premature atherosclerosis, tendon xanthomas, juvenile onset cataracts, osteoporosis, and mild pulmonary insufficiency. Long-term treatment with CDCA can result in amelioration of neurological symptoms and an improved prognosis.

\section{RETINAL DISEASES}

Bear bile has been used in Chinese medicine for several millennia to treat visual disorders (Boatright et al., 2006). This has led to a number of studies investigating the primary constituent of bear bile, TUDCA, as a potential therapeutic agent for several ophthalmological diseases (Boatright et al., 2009). TUDCA has been investigated in retinitis pigmentosa, a heterogeneous group of disorders of retinal degeneration in which progressive peripheral and night vision loss occurs, with central vision impairment. Mutations in about 200 genes, including the gene encoding rhodopsin (RHO), have been identified that cause apoptosis of photoreceptor cells (Daiger et al., 2013). The RHO $\mathrm{P} 23 \mathrm{H}$ mutation is the most common cause of retinitis pigmentosa in the United States that is thought to produce structurally altered folding with retention in the endoplasmic reticulum and resulting cytotoxicity. TUDCA administered intraperitoneally once a week from weaning until 4 months of age to homozygous $\mathrm{P} 23 \mathrm{H}$ line-3 rats reduced photoreceptor loss across the retina and preserved synapses between photoreceptors and bipolar or horizontal cells (Fernandez-Sanchez et al., 2011). In the $r d 10$ mouse model of retinitis pigmentosa, in which the mice carry a missense mutation in exon 7 of the Pde6b gene resulting in rod photoreceptor cell death within a month after birth, 
subcutaneous administration of TUDCA resulted in higher cone cell density in all 4 quadrants of the retina (Oveson et al., 2011). TUDCA also decreased apoptosis, preserved the normal retinal photoreceptor cellular architecture, and maintained amplitudes of dark-adapted electroretinogram a- and b-waves (Boatright et al., 2006), even at later stages of severe photoreceptor loss (Phillips et al., 2008).

Mutations in the retinoid isomerase (RPE65) or lecithinretinol acyltransferase (LRAT) genes underlie Leber congenital amaurosis (den Hollander et al., 2008). LRAT is involved in 11cis retinal recycling in the retinal pigment epithelium through mediating the esterification of all-trans retinol to all-trans retinyl esters, which are the substrates for RPE65. Homozygous Lrat knockout mice that were subcutaneously administered TUDCA every 3 days from 1 to 4 weeks after birth had about a threefold increase in cone density in the ventral and central retina and increased ER-associated protein degradation (Zhang et al., 2012; Fu and Zhang, 2014). Twice per week subcutaneous injections of TUDCA also preserved ERG b-waves and the outer nuclear layer of the retina in Bardet-Biedl syndrome type 1 mice $\left(B b s 1^{\mathrm{M} 390 \mathrm{R} / \mathrm{M} 390 \mathrm{R}}\right)$, another model for retinal degeneration (Drack et al., 2012).

TUDCA ameliorated cell death and loss of photoreceptor function after exposure to high levels of light (10,000 lux) that can induce retinal degeneration in albino Balb/C mice (Boatright et al., 2006). TUDCA pre-treatment of ex vivo perfused whole mount feline retinas repeatedly exposed to light stimulation and dark adaptations over $5 \mathrm{~h}$ resulted in greater receptive field size, decreased irradiance threshold, and maintenance of the contrast threshold (Xia et al., 2015). Retinal dystrophy can be caused by defects in the phagocytosis by retinal pigment epithelium cells of the photoreceptor outer segments that are shed, a continuous and extremely active process involving thousands of shed membranous disks each day. TUDCA was found to increase phagocytic activity and inhibit hydrogen peroxide induced impairment of phagocytosis by both cultured ARPE-19 cells and primary human retinal pigment epithelium cells (Murase et al., 2015). The phosphorylation of MerTK was significantly increased by TUDCA in a concentration-dependent manner but did not affect expression of the ER stress marker glucose regulated protein-78 (GRP-78).

Bile acids also had an effect in ameliorating cell death in a model of retinal detachment via intraperitoneal injection of TUDCA at 3 and 5 days post detachment, times when acute photoreceptor loss occurs in the outer nuclear layer of the retina (Mantopoulos et al., 2011). TUDCA treatment also blocked the production of protein carbonyls with decreased caspase activation but did not decrease endoplasmic reticulum (ER) stress. In a diabetic retinopathy model, TUDCA significantly decreased apoptosis measured by TUNEL assay of primary rat retinal neural cells exposed to $30 \mathrm{mM}$ glucose concentration, which causes caspase-independent cell death (Gaspar et al., 2013). TUDCA also decreased the amount of apoptosis-inducing factor (AIF) released from the mitochondria and its subsequent accumulation in the nucleus. Production of protein carbonyls and ROS were also significantly decreased after TUDCA treatment.
Over-stimulation of N-methyl-D-aspartate (NMDA) receptors, one of three ionotropic glutamate receptor subtypes that are expressed in inner retinal cells, by intravitreal injection of NMDA has frequently been used to model the cell death pathway that occurs in retinal diseases such as glaucoma (Danesh-Meyer, 2011). This model of excitotoxicity causes disruption of sodium-potassium balance, calcium overload, mitochondrial dysfunction, and oxidative stress (Gomez-Vicente et al., 2015). In mice, administration of TUDCA prior to the intravitreal injection of NMDA was found to increase survival of retinal ganglion cells.

\section{ACUTE STROKE, SPINAL CORD INJURY, AND NEUROPATHY}

A single intravenous (i.v.) dose of TUDCA administered to rats $60 \mathrm{~min}$ after occlusion of the middle cerebral artery markedly reduced infarct size and apoptosis and preserved mitochondrial integrity (Rodrigues et al., 2002). The single i.v. dose resulted in a significant increase in brain UDCA levels from near undetectable levels to $0.15 \mathrm{nmol} / \mathrm{g}$. TUDCA was also found to have an antiinflammatory effect in the context of the central nervous system. TUDCA reduced glial cell activation and microglial chemotaxis and reduced expression of the MCP-1 chemoattractant and vascular adhesion proteins such as VCAM-1 in microglial cells and astrocytes treated with either interferon gamma (IFN- $\gamma$ ) or lipopolysaccharide and IFN- $\gamma$ (Yanguas-Casás et al., 2014). Injection of TUDCA into the carotid artery $1 \mathrm{~h}$ prior to or up to $6 \mathrm{~h}$ after collagenase injection into the striatum to induce hemorrhagic lesions decreased lesion volumes, peri-hematoma apoptosis, and caspase activity at 2 days by about $50 \%$, as well as decreased NF- $\mathrm{B}$ and increased AKT activation that was associated with neurobehavioral improvement (Rodrigues et al., 2003). TUDCA injected intraperitoneally $1 \mathrm{~min}$ after spinal cord crush injury decreased apoptosis and corresponding tissue injury (Colak et al., 2008). In a phase II open-label study of oral doxycycline and TUDCA taken three times/day for 1 year in patients with transthyretin amyloidosis (ATTR), progression of neuropathy was stabilized (Obici et al., 2012).

\section{MODULATION OF NEUROTRANSMITTER ACTIVITY}

The wake period of normal mice was found to be increased with administration of UDCA that also decreased slow wave sleep (Yanovsky et al., 2012). In contrast, administration of UDCA to histidine decarboxylase knockout mice, that are deficient in histamine that stimulates arousal, decreased wakefulness and altered cortical EEG and sleep-wake parameters. Using in vitro patch-clamp recordings from histaminergic neurons, UDCA was found to inhibit GABAergic currents and to serve as an antagonist for $\mathrm{GABA}_{\mathrm{A}}$ receptors expressed in HEK293 cells. In one of the few studies to analyze more than one or two bile acids, a structure-function relationship analysis was performed using cultured hypothalamic neurons (Schubring et al., 2012). Bile acids were found to modulate firing frequency 
and synchrony, and to block activity of $\mathrm{GABA}_{\mathrm{A}}$ and NMDA receptors. Antagonist activity for both the $\mathrm{GABA}_{\mathrm{A}}$ and NMDA receptors was strongest for CDCA followed by $\mathrm{DCA}, \mathrm{CA}$, and dehydrocholate. Involvement of bile acid binding to TGR5 was excluded as a potential mechanism in the neurotransmitter receptor blockade (Keitel et al., 2010).

CA was found to serve as an inhibiter for $\mathrm{N}$-type $\mathrm{Ca}^{2+}$ channel currents of neurons isolated from the caudal paravertebral sympathetic ganglia of adult bull frogs (Rana catesbeiana), although open and shut times, slope conductance, and single channel current amplitude, were not significantly affected (Lee et al., 2012). Overstimulation of glutamate receptors to induce excitotoxicity in neurons isolated from late stage fetal rat brains was largely suppressed by GUDCA. This bile acid appeared to suppress glutamate release in either normal or microglia-depleted hippocampal tissue slices (Silva et al., 2012). Pretreatment of primary cultures of fetal rat cortical neurons with TUDCA significantly reduced glutamate excitotoxicity associated cell death. TUDCA treatment resulted in phosphorylation and translocation of the pro-apoptotic Bad protein from the mitochondria to the cytosol. Inhibiting the PI3K signaling pathway blocked the anti-apoptotic effects of TUDCA (Castro et al., 2004).

\section{POTENTIAL AS NEUROTROPHIC FACTORS}

Neurotrophic factors are classified primarily as proteins that bind to receptors, e.g., tropomyosin related kinase receptors TrkA, TrkB, and TrkC and p75 neurotrophin receptor (p75NTR) that can modulate Trk activation, to activate signaling pathways that promote neuronal growth and survival (Rodrigues et al., 2014). Several molecules have been identified as neurotrophic factors, including brain-derived neurotrophic factor (BDNF), ciliary neurotrophic factor (CNTF), and glial cell-line-derived neurotrophic factor (GDNF) that can stimulate the growth and differentiation of neuronal cells, as well as prevent cell death. Despite the therapeutic promise of such neurotrophic factors for treating neurodegenerative disorders (Tovar-Y-Romo et al., 2014), their limited bioavailability presents a major roadblock. Mimetics with more favorable bioavailability profiles

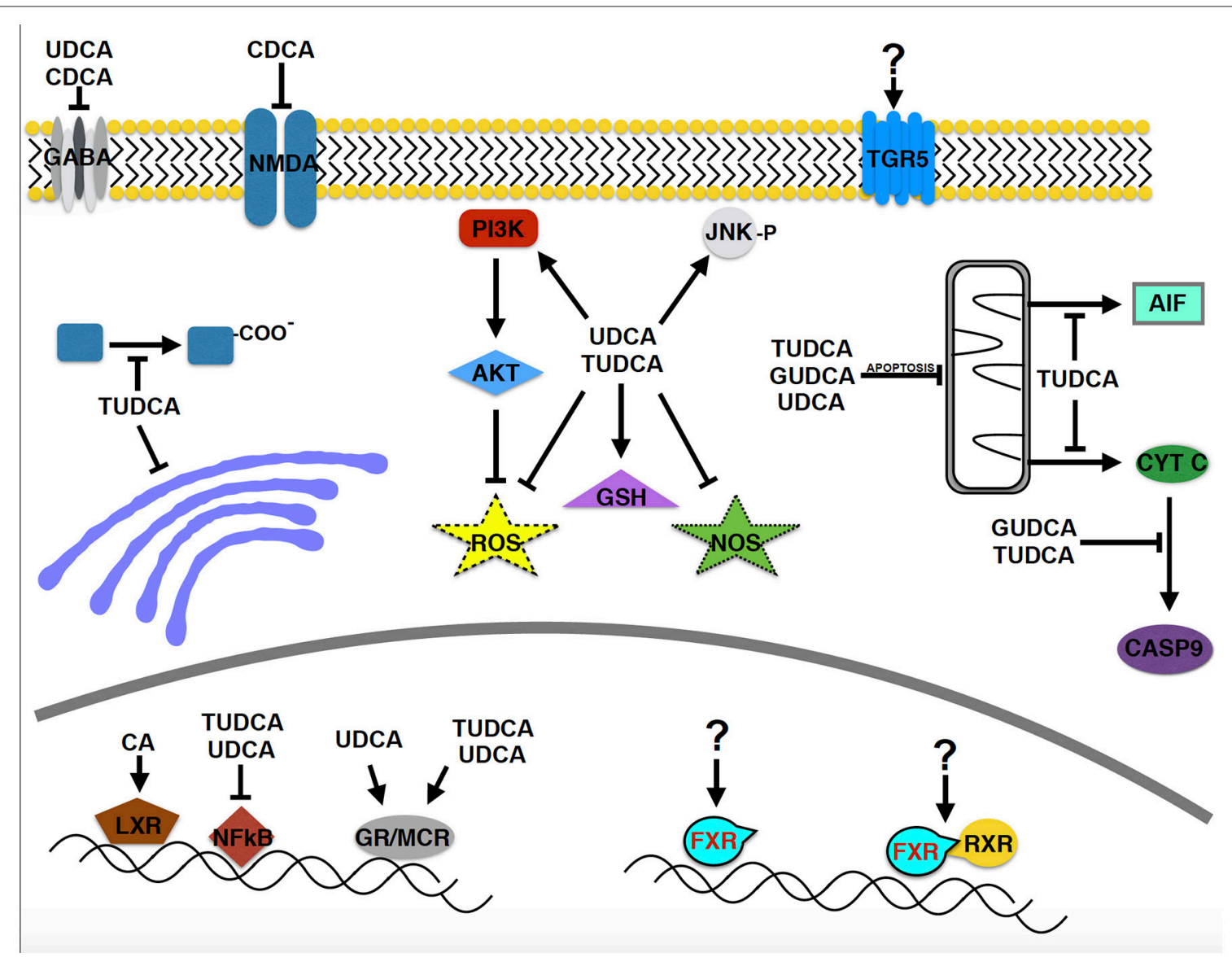

FIGURE 2 | Molecular pathways implicated in the neuroprotective effects of bile acids in neurodegenerative disease models. Despite the relatively large structurally related group of bile acids, relatively few have been studied in neurodegenerative disorders. A major focus has been on apoptotic pathways and the PI3 kinase and AKT signaling pathway. Surprisingly, the primary signaling pathways through which bile acids act, TGR5, FXR, and FXR/RXR have received essentially no attention (designated by "?"), despite that retinoic acid is known to be a potent neurotrophic molecule. 
have therefore been pursued (Tiwari and Chaturvedi, 2014), as well as investigation of proneurogenic peptides and small molecules that exhibit neuroprotective properties (Airavaara et al., 2012), including retinoic acid (Malik et al., 2000; Riancho et al., 2016). Retinoic acid shares a common role in regulating lipid homeostasis with bile acids, whose cognate receptor FXR can form heterodimers with $\mathrm{RXR} \alpha$. Thus, retinoic acid may activate the FXR signaling pathway and vice versa (Yang et al., 2014). Whether bile acids mediate effects in the nervous system through a similar mechanism is not known (Figure 1).

\section{CONCLUSIONS AND OUTLOOK}

Neuroprotective effects of several bile acids are well documented in a wide range of neurodegenerative diseases, including $\mathrm{AD}, \mathrm{PD}$, ALS, HD, and retinal degeneration, in cellular and animal models and in human clinical trials. Due to the historical precedent set by the use of bear bile in ancient Chinese medicine, the majority of the studies have focused on UDCA and its derivatives. However, bile acids are a relatively large group of structurally related molecules, thus little is known about the potential efficacy of other bile acid species or the roles of circulating vs.

\section{REFERENCES}

Airavaara, M., Voutilainen, M. H., Wang, Y., and Hoffer, B. (2012). Neurorestoration. Parkinsonism Relat. Disord. 18(Suppl. 1), S143-S146. doi: 10.1016/S1353-8020(11)70045-1

Andersson, S., Gustafsson, N., Warner, M., and Gustafsson, J. A. (2005). Inactivation of liver $\mathrm{X}$ receptor beta leads to adult-onset motor neuron degeneration in male mice. Proc. Natl. Acad. Sci. U.S.A. 102, 3857-3862. doi: 10. 1073/pnas.0500634102

Bjorkhem, I. (2013). Cerebrotendinous xanthomatosis. Curr. Opin. Lipidol. 24, 283-287. doi: 10.1097/MOL.0b013e328362df13

Björkhem, I., and Hansson, M. (2010). Cerebrotendinous xanthomatosis: an inborn error in bile acid synthesis with defined mutations but still a challenge. Biochem. Biophys. Res. Commun. 396, 46-49. doi: 10.1016/j.bbrc.2010.02.140

Boatright, J. H., Moring, A. G., McElroy, C., Phillips, M. J., Do, V. T., Chang, B., et al. (2006). Tool from ancient pharmacopoeia prevents vision loss. Mol. Vis. $12,1706-1714$

Boatright, J. H., Nickerson, J. M., Moring, A. G., and Pardue, M. T. (2009). Bile acids in treatment of ocular disease. J. Ocul. Biol. Dis. Infor. 2, 149-159. doi: 10. 1007/s12177-009-9030-x

Carvalho, I. M., Coelho, P. B., Costa, P. C., Marques, C. S., Oliveira, R. S., and Ferreira, D. C. (2015). Current neurogenic and neuroprotective strategies to prevent and treat neurodegenerative and neuropsychiatric disorders. Neuromol. Med. 17, 404-422. doi: 10.1007/s12017-015-8369-3

Castro, R. E., Solá, S., Ramalho, R. M., Steer, C. J., and Rodrigues, C. M. (2004). The bile acid tauroursodeoxycholic acid modulates phosphorylation and translocation of bad via phosphatidylinositol 3-kinase in glutamate-induced apoptosis of rat cortical neurons. J. Pharmacol. Exp. Ther. 311, 845-852. doi: 10. 1124/jpet.104.070532

Castro-Caldas, M., Carvalho, A. N., Rodrigues, E., Henderson, C. J., Wolf, C. R., Rodrigues, C. M., et al. (2012). Tauroursodeoxycholic acid prevents MPTPinduced dopaminergic cell death in a mouse model of Parkinson's disease. Mol. Neurobiol. 46, 475-486. doi: 10.1007/s12035-012-8295-4

Cheng, J. B., Jacquemin, E., Gerhardt, M., Nazer, H., Cresteil, D., Heubi, J. E., et al. (2003). Molecular genetics of 3beta-hydroxy-Delta5-C27-steroid oxidoreductase deficiency in 16 patients with loss of bile acid synthesis and liver disease. J. Clin. Endocrinol. Metab. 88, 1833-1841. doi: 10.1210/jc.2002-021580 endogenous bile acids synthesized within the central nervous system. In addition, most of the mechanistic studies have been centered on apoptosis and related pathways (Figure 2). However, essentially no data is available on the primary signaling pathways through which bile acids act, the cellular receptor TGR5 and the nuclear receptors FXR and RXR $\alpha$, despite the well-known function of retinoic acid as a potent neurotrophic molecule. Determining the precise molecular mechanism(s) of neuroprotection by bile acids in neurodegenerative disorders will be important to realize their future therapeutic potential.

\section{AUTHOR CONTRIBUTIONS}

GG and HA designed, wrote, revised, and edited the manuscript. Both authors approved the submitted version of the manuscript.

\section{FUNDING}

This work was funded by the Lewis Katz School of Medicine at Temple University Department of Medical Genetics and Molecular Biochemistry and the Joseph \& Rebecca Goodfriend Endowed Chair in Genetics.

Chun, H. S., and Low, W. C. (2012). Ursodeoxycholic acid suppresses mitochondria-dependent programmed cell death induced by sodium nitroprusside in SH-SY5Y cells. Toxicology 292, 105-112. doi: 10.1016/j. tox.2011.11.020

Colak, A., Kelten, B., Sagmanligil, A., Akdemir, O., Karaoglan, A., Sahan, E., et al. (2008). Tauroursodeoxycholic acid and secondary damage after spinal cord injury in rats. J. Clin. Neurosci. 15, 665-671. doi: 10.1016/j.jocn.2007.06.002

Cortez, L. M., Campeau, J., Norman, G., Kalayil, M., Van der Merwe, J., McKenzie, D., et al. (2015). Bile acids reduce prion conversion, reduce neuronal loss, and prolong male survival in models of prion disease. J. Virol. 89, 7660-7672. doi: 10.1128/JVI.01165-15

Daiger, S. P., Sullivan, L. S., and Bowne, S. J. (2013). Genes and mutations causing retinitis pigmentosa. Clin. Genet. 84, 132-141. doi: 10.1111/cge.12203

Danesh-Meyer, H. V. (2011). Neuroprotection in glaucoma: recent and future directions. Curr. Opin. Ophthalmol. 22, 78-86. doi: 10.1097/ICU. ob013e32834372ec

Davies, S. W., Turmaine, M., Cozens, B. A., DiFiglia, M., Sharp, A. H., Ross, C. A., et al. (1997). Formation of neuronal intranuclear inclusions underlies the neurological dysfunction in mice transgenic for the HD mutation. Cell 90, 537-548. doi: 10.1016/S0092-8674(00)80513-9

den Hollander, A. I., Roepman, R., Koenekoop, R. K., and Cremers, F. P. (2008). Leber congenital amaurosis: genes, proteins and disease mechanisms. Prog. Retin. Eye Res. 27, 391-419. doi: 10.1016/j.preteyeres.2008.05.003

Diekstra, F. P., Saris, C. G., van Rheenen, W., Franke, L., Jansen, R. C., van Es, M. A., et al. (2012). Mapping of gene expression reveals CYP27A1 as a susceptibility gene for sporadic ALS. PLoS ONE 7:e35333. doi: 10.1371/journal. pone. 0035333

Drack, A. V., Dumitrescu, A. V., Bhattarai, S., Gratie, D., Stone, E. M., Mullins, R., et al. (2012). TUDCA slows retinal degeneration in two different mouse models of retinitis pigmentosa and prevents obesity in Bardet-Biedl syndrome type 1 mice. Invest. Ophthalmol. Vis. Sci. 53, 100-106. doi: 10.1167/iovs.11-8544

Elia, A. E., Lalli, S., Monsurró, M. R., Sagnelli, A., Taiello, A. C., Reggiori, B., et al. (2016). Tauroursodeoxycholic acid in the treatment of patients with amyotrophic lateral sclerosis. Eur. J. Neurol. 23, 45-52. doi: 10.1111/ene.12664

Fernandez-Sanchez, L., Lax, P., Pinilla, I., Martín-Nieto, J., and Cuenca, N. (2011). Tauroursodeoxycholic acid prevents retinal degeneration in transgenic $\mathrm{P} 23 \mathrm{H}$ rats. Invest. Ophthalmol. Vis. Sci. 52, 4998-5008. doi: 10.1167/iovs.11-7496 
Fu, Y., and Zhang, T. (2014). Pathophysilogical mechanism and treatment strategies for Leber congenital amaurosis. Adv. Exp. Med. Biol. 801, 791-796. doi: 10.1007/978-1-4614-3209-8_99

Garcia-Segura, L. M., and Balthazart, J. (2009). Steroids and neuroprotection: new advances. Front. Neuroendocrinol. 30:v-ix. doi: 10.1016/j.yfrne.2009.04.006

Gaspar, J. M., Martins, A., Cruz, R., Rodrigues, C. M., Ambrósio, A. F., and Santiago, A. R. (2013). Tauroursodeoxycholic acid protects retinal neural cells from cell death induced by prolonged exposure to elevated glucose. Neuroscience 253, 380-388. doi: 10.1016/j.neuroscience.2013.08.053

Ghanbari, M., Darweesh, S. K., de Looper, H. W., van Luijn, M. M., Hofman, A., Ikram, M. A., et al. (2016). Genetic variants in microRNAs and their binding sites are associated with the risk of Parkinson disease. Hum. Mutat. 37, 292-300. doi: 10.1002/humu.22943

Gómez-Vicente, V., Lax, P., Fernandez-Sanchez, L., Rondon, N., Esquiva, G., Germain, F., et al. (2015). Neuroprotective effect of tauroursodeoxycholic acid on N-methyl-D-aspartate-induced retinal ganglion cell degeneration. PLoS ONE 10:e0137826. doi: 10.1371/journal.pone.0137826

Hodge, R. J., and Nunez, D. J. (2016). Therapeutic potential of Takeda-G-proteinreceptor-5 (TGR5) agonists. Hope or hype? Diabetes Obes. Metab. 18, 439-443. doi: $10.1111 /$ dom. 12636

Hoeke, M. O., Heegsma, J., Hoekstra, M., Moshage, H., and Faber, K. N. (2014). Human FXR regulates SHP expression through direct binding to an LRH-1 binding site, independent of an IR-1 and LRH-1. PLOS ONE 9:e88011. doi: 10. 1371/journal.pone.0088011

Huang, F., Wang, T., Lan, Y., Yang, L., Pan, W., Zhu, Y., et al. (2015). Deletion of mouse FXR gene disturbs multiple neurotransmitter systems and alters neurobehavior. Front. Behav. Neurosci. 9:70. doi: 10.3389/fnbeh.2015.00070

Huang, Q., Du, X., He, X., Yu, Q., Hu, K., Breitwieser, W., et al. (2016). JNKmediated activation of ATF2 contributes to dopaminergic neurodegeneration in the MPTP mouse model of Parkinson's disease. Exp. Neurol. 277, 296-304. doi: 10.1016/j.expneurol.2015.10.010

Imran, M., and Mahmood, S. (2011). An overview of human prion diseases. Virol. J. 8, 559. doi: 10.1186/1743-422X-8-559

Joo, S. S., Won, T. J., and Lee, D. I. (2004). Potential role of ursodeoxycholic acid in suppression of nuclear factor kappa B in microglial cell line (BV-2). Arch. Pharm. Res. 27, 954-960. doi: 10.1007/BF02975850

Kaemmerer, W. F., Rodrigues, C. M., Steer, C. J., and Low, W. C. (2001). Creatinesupplemented diet extends Purkinje cell survival in spinocerebellar ataxia type 1 transgenic mice but does not prevent the ataxic phenotype. Neuroscience 103, 713-724. doi: 10.1016/S0306-4522(01)00017-3

Keene, C. D., Rodrigues, C. M., Eich, T., Chhabra, M. S., Steer, C. J., and Low, W. C. (2002). Tauroursodeoxycholic acid, a bile acid, is neuroprotective in a transgenic animal model of Huntington's disease. Proc. Natl. Acad. Sci. U.S.A. 99, 10671-10676. doi: 10.1073/pnas.162362299

Keene, C. D., Rodrigues, C. M., Eich, T., Linehan-Stieers, C., Abt, A., Kren, B. T., et al. (2001). A bile acid protects against motor and cognitive deficits and reduces striatal degeneration in the 3-nitropropionic acid model of Huntington's disease. Exp. Neurol. 171, 351-360. doi: 10.1006/exnr.2001.7755

Keitel, V., Görg, B., Bidmon, H. J., Zemtsova, I., Spomer, L., Zilles, K., et al. (2010). The bile acid receptor TGR5 (Gpbar-1) acts as a neurosteroid receptor in brain. Glia 58, 1794-1805. doi: 10.1002/glia.21049

Koster, K. P., Smith, C., Valencia-Olvera, A. C., Thatcher, G. R. J., Tai, L. M., and $\mathrm{LaDu}, \mathrm{M} . \mathrm{J}$. (2016). Rexinoids as therapeutics for Alzheimer disease: role of APOE. Curr. Top. Med. Chem. doi: 10.2174/1568026616666160617090227. [Epub ahead of print].

Lee, H. K., Lee, K. H., and Cho, E. S. (2012). Bile acid inhibition of Ntype calcium channel currents from sympathetic ganglion neurons. Korean $\mathrm{J}$. Physiol. Pharmacol. 16, 25-30. doi: 10.4196/kjpp.2012.16.1.25

Lieu, T., Jayaweera, G., and Bunnett, N. W. (2014). GPBA: a GPCR for bile acids and an emerging therapeutic target for disorders of digestion and sensation. $\mathrm{Br}$. J. Pharmacol. 171, 1156-1166. doi: 10.1111/bph.12426

Lo, A. C., Callaerts-Vegh, Z., Nunes, A. F., Rodrigues, C. M., and D'Hooge, R. (2013). Tauroursodeoxycholic acid (TUDCA) supplementation prevents cognitive impairment and amyloid deposition in APP/PS1 mice. Neurobiol. Dis. 50, 21-29. doi: 10.1016/j.nbd.2012.09.003

Luo, Y., Hoffer, A., Hoffer, B., and Qi, X. (2015). Mitochondria: a therapeutic target for Parkinson's disease? Int. J. Mol. Sci. 16, 20704-20730. doi: 10.3390/ ijms160920704
Maier, O., Böhm, J., Dahm, M., Brück, S., Beyer, C., and Johann, S. (2013). Differentiated NSC-34 motoneuron-like cells as experimental model for cholinergic neurodegeneration. Neurochem. Int. 62, 1029-1038. doi: 10.1016/ j.neuint.2013.03.008

Malik, M. A., Blusztajn, J. K., and Greenwood, C. E. (2000). Nutrients as trophic factors in neurons and the central nervous system: role of retinoic acid. J. Nutr. Biochem. 11, 2-13. doi: 10.1016/S0955-2863(99)00066-2

Mangiarini, L., Sathasivam, K., Seller, M., Cozens, B., Harper, A., Hetherington, C., et al. (1996). Exon 1 of the HD gene with an expanded CAG repeat is sufficient to cause a progressive neurological phenotype in transgenic mice. Cell 87, 493-506. doi: 10.1016/S0092-8674(00)81369-0

Mantopoulos, D., Murakami, Y., Comander, J., Thanos, A., Roh, M., Miller, J. W., et al. (2011). Tauroursodeoxycholic acid (TUDCA) protects photoreceptors from cell death after experimental retinal detachment. PLoS ONE 6:e24245. doi: 10.1371/journal.pone. 0024245

Mapstone, M., Cheema, A. K., Fiandaca, M. S., Zhong, X., Mhyre, T. R., MacArthur, L. H., et al. (2014). Plasma phospholipids identify antecedent memory impairment in older adults. Nat. Med. 20, 415-418. doi: 10.1038/nm. 3466

Meera, P., Pulst, S. M., and Otis, T. S. (2016). Cellular and circuit mechanisms underlying spinocerebellar ataxias. J. Physiol. 594, 4653-4660. doi: 10.1113/ JP271897

Min, J. H., Hong, Y. H., Sung, J. J., Kim, S. M., Lee, J. B., and Lee, K. W. (2012). Oral solubilized ursodeoxycholic acid therapy in amyotrophic lateral sclerosis: a randomized cross-over trial. J. Korean Med. Sci. 27, 200-206. doi: 10.3346/ jkms.2012.27.2.200

Mortiboys, H., Aasly, J., and Bandmann, O. (2013). Ursocholanic acid rescues mitochondrial function in common forms of familial Parkinson's disease. Brain 136(Pt 10), 3038-3050. doi: 10.1093/brain/awt224

Murase, H., Tsuruma, K., Shimazawa, M., and Hara, H. (2015). TUDCA promotes phagocytosis by retinal pigment epithelium via MerTK activation. Invest. Ophthalmol. Vis. Sci. 56, 2511-2518. doi: 10.1167/iovs.14-15962

Nunes, A. F., Amaral, J. D., Lo, A. C., Fonseca, M. B., Viana, R. J., Callaerts-Vegh, Z., et al. (2012). TUDCA, a bile acid, attenuates amyloid precursor protein processing and amyloid-beta deposition in APP/PS1 mice. Mol. Neurobiol. 45, 440-454. doi: 10.1007/s12035-012-8256-y

Obici, L., Cortese, A., Lozza, A., Lucchetti, J., Gobbi, M., Palladini, G., et al. (2012). Doxycycline plus tauroursodeoxycholic acid for transthyretin amyloidosis: a phase II study. Amyloid 19(Suppl. 1), 34-36. doi: 10.3109/13506129.2012. 678508

Oveson, B. C., Iwase, T., Hackett, S. F., Lee, S. Y., Usui, S., Sedlak, T. W., et al. (2011). Constituents of bile, bilirubin and TUDCA, protect against oxidative stress-induced retinal degeneration. J. Neurochem. 116, 144-153. doi: 10.1111/ j.1471-4159.2010.07092.x

Parry, G. J., Rodrigues, C. M., Aranha, M. M., Hilbert, S. J., Davey, C., Kelkar, P., et al. (2010). Safety, tolerability, and cerebrospinal fluid penetration of ursodeoxycholic Acid in patients with amyotrophic lateral sclerosis. Clin. Neuropharmacol. 33, 17-21. doi: 10.1097/WNF.0b013e3181c47569

Phillips, M. J., Walker, T. A., Choi, H. Y., Faulkner, A. E., Kim, M. K., Sidney, S. S., et al. (2008). Tauroursodeoxycholic acid preservation of photoreceptor structure and function in the rd10 mouse through postnatal day 30. Invest. Ophthalmol. Vis. Sci. 49, 2148-2155. doi: 10.1167/iovs.07-1012

Prawitt, J., Caron, S., and Staels, B. (2011). Bile acid metabolism and the pathogenesis of type 2 diabetes. Curr. Diab. Rep. 11, 160-166. doi: 10.1007/ s11892-011-0187-x

Ramalho, R. M., Nunes, A. F., Dias, R. B., Amaral, J. D., Lo, A. C., D'Hooge, R., et al. (2013). Tauroursodeoxycholic acid suppresses amyloid beta-induced synaptic toxicity in vitro and in APP/PS1 mice. Neurobiol. Aging 34, 551-561. doi: 10. 1016/j.neurobiolaging.2012.04.018

Riancho, J., Berciano, M. T., Ruiz-Soto, M., Berciano, J., Landreth, G., and Lafarga, M. (2016). Retinoids and motor neuron disease: potential role in amyotrophic lateral sclerosis. J. Neurol. Sci. 360, 115-120. doi: 10.1016/j.jns.2015.11.058

Rodrigues, C. M., Sola, S., Nan, Z., Castro, R. E., Ribeiro, P. S., Low, W. C., et al. (2003). Tauroursodeoxycholic acid reduces apoptosis and protects against neurological injury after acute hemorrhagic stroke in rats. Proc. Natl. Acad. Sci. U.S.A. 100, 6087-6092. doi: 10.1073/pnas. 1031632100

Rodrigues, C. M., Spellman, S. R., Solá, S., Grande, A. W., Linehan-Stieers, C., Low, W. C., et al. (2002). Neuroprotection by a bile acid in an acute stroke model 
in the rat. J. Cereb. Blood Flow Metab. 22, 463-471. doi: 10.1097/00004647200204000-00010

Rodrigues, C. M., Stieers, C. L., Keene, C. D., Ma, X., Kren, B. T., Low, W. C., et al. (2000). Tauroursodeoxycholic acid partially prevents apoptosis induced by $3-$ nitropropionic acid: evidence for a mitochondrial pathway independent of the permeability transition. J. Neurochem. 75, 2368-2379. doi: 10.1046/j.1471-4159. 2000.0752368.x

Rodrigues, T. M., Jeronimo-Santos, A., Outeiro, T. F., Sebastião, A. M., and Diógenes, M. J. (2014). Challenges and promises in the development of neurotrophic factor-based therapies for Parkinson's disease. Drugs Aging 31, 239-261. doi: 10.1007/s40266-014-0160-x

Ross, C. A., Aylward, E. H., Wild, E. J., Langbehn, D. R., Long, J. D., Warner, J. H., et al. (2014). Huntington disease: natural history, biomarkers and prospects for therapeutics. Nat. Rev. Neurol. 10, 204-216. doi: 10.1038/nrneurol.2014.24

Scheltens, P., Blennow, K., Breteler, M. M. B., de Strooper, B., Frisoni, G. B., Salloway, S., et al. (2016). Alzheimer's disease. Lancet 388, 505-517. doi: 10. 1016/S0140-6736(15)01124-1

Schonewille, M., de Boer, J. F., and Groen, A. K. (2016). Bile salts in control of lipid metabolism. Curr. Opin. Lipidol. 27, 295-301. doi: 10.1097/MOL. 0000000000000303

Schubring, S. R., Fleischer, W., Lin, J. S., Haas, H. L., and Sergeeva, O. A. (2012). The bile steroid chenodeoxycholate is a potent antagonist at NMDA and GABAA $_{\text {A }}$ receptors. Neurosci. Lett. 506, 322-326. doi: 10.1016/j.neulet.2011.11. 036

Silva, S. L., Vaz, A. R., Diógenes, M. J., van Rooijen, N., Sebastião, A. M., Fernandes, A., et al. (2012). Neuritic growth impairment and cell death by unconjugated bilirubin is mediated by NO and glutamate, modulated by microglia, and prevented by glycoursodeoxycholic acid and interleukin-10. Neuropharmacology 62, 2398-2408. doi: 10.1016/j.neuropharm.2012.02.002

Solá, S., Amaral, J. D., Borralho, P. M., Ramalho, R. M., Castro, R. E., Aranha, M. M., et al. (2006). Functional modulation of nuclear steroid receptors by tauroursodeoxycholic acid reduces amyloid beta-peptide-induced apoptosis. Mol. Endocrinol. 20, 2292-2303. doi: 10.1210/me.2006-0063

Solá, S., Castro, R. E., Laires, P. A., Steer, C. J., and Rodrigues, C. M. (2003). Tauroursodeoxycholic acid prevents amyloid-beta peptide-induced neuronal death via a phosphatidylinositol 3-kinase-dependent signaling pathway. Mol. Med. 9, 226-234. doi: 10.2119/2003-00042.Rodrigues

Song, G. G., and Lee, Y. H. (2013). Pathway analysis of genome-wide association studies for Parkinson's disease. Mol. Biol. Rep. 40, 2599-2607. doi: 10.1007/ s11033-012-2346-9

Theofilopoulos, S., Wang, Y., Kitambi, S. S., Sacchetti, P., Sousa, K. M., Bodin, K., et al. (2013). Brain endogenous liver X receptor ligands selectively promote midbrain neurogenesis. Nat. Chem. Biol. 9, 126-133. doi: 10.1038/nchembio. 1156

Tiwari, S. K., and Chaturvedi, R. K. (2014). Peptide therapeutics in neurodegenerative disorders. Curr. Med. Chem. 21, 2610-2631. doi: 10. $2174 / 0929867321666140217125857$
Tovar-Y-Romo, L. B., Ramírez-Jarquin, U. N., Lazo-Gómez, R., and Tapia, R. (2014). Trophic factors as modulators of motor neuron physiology and survival: implications for ALS therapy. Front. Cell. Neurosci. 8:61. doi: 10.3389/fncel. 2014.00061

Vaz, A. R., Cunha, C., Gomes, C., Schmucki, N., Barbosa, M., and Brites, D. (2015). Glycoursodeoxycholic acid reduces matrix metalloproteinase-9 and caspase- 9 activation in a cellular model of superoxide dismutase-1 neurodegeneration. Mol. Neurobiol. 51, 864-877. doi: 10.1007/s12035-014$8731-8$

Veyrat-Durebex, C., Corcia, P., Dangoumau, A., Laumonnier, F., Piver, E., Gordon, P. H., et al. (2014). Advances in cellular models to explore the pathophysiology of amyotrophic lateral sclerosis. Mol. Neurobiol. 49, 966-983. doi: 10.1007/ s12035-013-8573-9

Windl, O., and Dawson, M. (2012). Animal prion diseases. Subcell. Biochem. 65, 497-516. doi: 10.1007/978-94-007-5416-4_18

Xia, H., Nan, Y., Huang, X., Gao, J., and Pu, M. (2015). Effects of tauroursodeoxycholic acid and alpha-lipoic-acid on the visual response properties of cat retinal ganglion cells: an in vitro study. Invest. Ophthalmol. Vis. Sci. 56, 6638-6645. doi: 10.1167/iovs.15-17301

Yang, F., He, Y., Liu, H. X., Tsuei, J., Jiang, X., Yang, L., et al. (2014). All-trans retinoic acid regulates hepatic bile acid homeostasis. Biochem. Pharmacol. 91, 483-489. doi: 10.1016/j.bcp.2014.08.018

Yanguas-Casás, N., Barreda-Manso, M. A., Nieto-Sampedro, M., and RomeroRamírez, L. (2014). Tauroursodeoxycholic acid reduces glial cell activation in an animal model of acute neuroinflammation. J. Neuroinflammation 11:50. doi: 10. 1186/1742-2094-11-50

Yanovsky, Y., Schubring, S. R., Yao, Q., Zhao, Y., Li, S., May, A., et al. (2012). Waking action of ursodeoxycholic acid (UDCA) involves histamine and GABAA receptor block. PLoS ONE 7:e42512. doi: 10.1371/journal.pone. 0042512

Zhang, J., Akwa, Y., el-Etr, M., Baulieu, E. E., and Sjövall, J. (1997). Metabolism of 27-, 25- and 24-hydroxycholesterol in rat glial cells and neurons. Biochem. J. 322 (Pt 1), 175-184.

Zhang, T., Baehr, W., and Fu, Y. (2012). Chemical chaperone TUDCA preserves cone photoreceptors in a mouse model of Leber congenital amaurosis. Invest. Ophthalmol. Vis. Sci. 53, 3349-3356. doi: 10.1167/iovs.12-9851

Conflict of Interest Statement: The authors declare that the research was conducted in the absence of any commercial or financial relationships that could be construed as a potential conflict of interest.

Copyright () 2016 Ackerman and Gerhard. This is an open-access article distributed under the terms of the Creative Commons Attribution License (CC BY). The use, distribution or reproduction in other forums is permitted, provided the original author(s) or licensor are credited and that the original publication in this journal is cited, in accordance with accepted academic practice. No use, distribution or reproduction is permitted which does not comply with these terms. 